\title{
Assessment of p16 and Ki67 Immunohistochemistry Expression in Squamous Intraepithelial Lesion with Cytohistomorphological Correlation
}

\author{
Apurv Ghosh ${ }^{1}$, Nirupama M*1, Nandan Padmanabha1(D), Hema Kini' ${ }^{1(D)}$ \\ 1. Department of Pathology, Kasturba Medical College, Mangalore, Manipal Academy of Higher Education, Manipal, India
}

\begin{tabular}{|c|c|}
\hline KEYWORDS & ABSTRACT \\
\hline 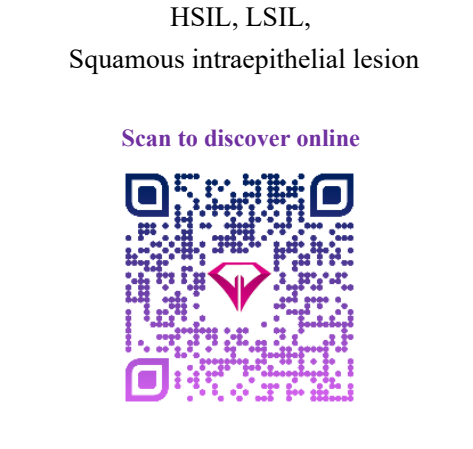 & $\begin{array}{l}\text { Background \& Objective: Cervical cancer is the most common cancer in women } \\
\text { worldwide with high mortality, necessitating quicker diagnostic methods. We wish to } \\
\text { enhance the existing cervical biopsies of Squamous Intraepithelial Lesions (SIL) using } \\
\text { p16 and Ki67 as surrogate markers to assess correlation between its positivity and } \\
\text { histological grade of the lesion. } \\
\text { Methods: Analysis of p16 and Ki67 expression was done on } 31 \text { histopathologically diagnosed } \\
\text { cases of SILs. Positive expression of p16 was assessed based on a scoring system and compared } \\
\text { with histology and cytology. Ki67 expression was studied and the correlation was observed } \\
\text { with degree of dysplasia. Twenty cases of chronic cervicitis was assigned to the control group } \\
\text { for comparison. }\end{array}$ \\
\hline $\begin{array}{c}\begin{array}{c}\text { Main Subjects: } \\
\text { GI pathology }\end{array} \\
\text { Received 03 Aug 2019; } \\
\text { Accepted 01 June 2020; }\end{array}$ & $\begin{array}{l}\text { Results: Cases of HSIL showed greater expression of p16 as compared to LSIL. } \\
\text { Sensitivity of p } 16 \text { for HSIL was higher than that for LSIL. The specificity for HSIL and } \\
\text { LSIL was } 100 \% \text {. Ki67 expression correlated well with the degree and level of dysplasia } \\
\text { with a significant P-value of } 0.002 \text {. }\end{array}$ \\
\hline doi $\underline{10.30699 / i j p .2020 .112421 .2208}$ & $\begin{array}{l}\text { Conclusion: p16 and Ki67 positivity of SILs should point towards further evaluation. The } \\
\text { expressions of p16 and Ki67 are useful markers for confirmation of SILs and in predicting } \\
\text { HPV infection which can be further confirmed by HPV DNA testing. }\end{array}$ \\
\hline
\end{tabular}

Corresponding Information: ～Nirupama M; Department of Pathology, Kasturba Medical College, Mangalore, India. Email: deptofpath@gmail.com

Copyright (C) 2020. This is an open-access article distributed under the terms of the Creative Commons Attribution- 4.0 International License which permits Share, copy and redistribution of the material in any medium or format or adapt, remix, transform, and build upon the material for any purpose, even commercially.

\section{Introduction}

Carcinoma cervix is one of the most common cancers among women worldwide and is the second most frequent type of cancer among Indian women (1). In India, an estimated 67,477 deaths have been attributed to cervical cancer (2). Early detection of the precursor lesions is therefore of paramount importance to reduce the mortality burden of the disease (3). Various screening modalities are being used to identify high risk patients and guide follow up and further management (4). The Papanicolaou (pap) cervical cytology test has been routinely used since 1960 to screen precursor lesions of the cervix and the diagnostic criteria have been updated since the introduction of the Bethesda system of reporting cervical cytology (5).

The earlier terminology of cervical intraepithelial neoplasia (CIN) and its categorisation into three groups (CIN 1,2,3) were riddled with interobserver variability (6$8)$. With the introduction of the two-tiered system to classify precursor lesions as squamous intraepithelial lesion (SIL), high grade or low grade reproducibility improved, but the quest to improve diagnostic accuracy has led to research with various immunohistochemical markers that target the basic mechanism in the pathogenesis of premalignant lesions of the cervix $(9,10)$ The Human papilloma virus (HPV) infection is responsible for SIL and its progression to invasive carcinoma has been well established. The high-risk 'HPV types 16 and 18' account for the majority of the women being affected (11). In the life cycle of the virus, the expression of oncoproteins E6 and E7 during the "transformation" phase, leads to inhibition of the tumour suppressor proteins $p 53$ and $R b$ gene. Overproduction of E2F leads to cyclin D1 inhibition and ultimately leading to p16 overexpression in the infected cells (12).

The overexpressed p16 can be detected using immunohistochemistry (IHC) and used in improving diagnostic accuracy (12-14). Another such marker is $\mathrm{Ki67}$, which is expressed during active phases of the cell cycle indicating cellular proliferation $(12,15)$. Over the years, a number of markers have been studied alone or in combination to best analyse, precursor lesions of the cervix to improve diagnostic accuracy $(9,16,17)$.

In resource poor centres which cater to an economically challenged population, the feasibility of running an entire battery of markers may not be possible 
$(10,18)$. In this study immunohistochemical expression of p16 and Ki67 were analysed in histologically and cytologically diagnosed cases of squamous intraepithelial lesions, and to correlate its association with high and lowgrade lesions.

\section{Materials and Methods \\ Case Selection}

A retrospective study was conducted between January 2015 and April 2017. Thirty-one cases were selected with histopathological diagnosis of cervical intraepithelial lesion (CIN 1,2,3) or squamous intraepithelial lesion (HSIL or LSIL). The specimens included hysterectomy specimens, conisation or punch biopsy samples. Tissue blocks with inadequate material, excessive haemorrhage or necrosis were excluded. The Pap smears were obtained for the cases wherever possible. Histopathological examination was performed at the Department of Pathology, at a tertiary centre in coastal region of India. All the H\&E slides were reviewed and a diagnosis of either high grade squamous intraepithelial lesion or low grade squamous intraepithelial lesion was assigned, based on the WHO criteria. The corresponding Pap cervical cytology smears were also reviewed wherever possible and the diagnosis was given, according to the Bethesda System of reporting cervical cytology. Twenty cases of histopathologically diagnosed cases of chronic cervicitis were taken as control for p16 and Ki67 immunostaining.

\section{Immunohistochemistry}

The representative H\&E section was selected for immunohistochemistry with p16 and Ki67. IHC was performed on formalin-fixed, paraffin-embedded tissue. Counterstaining was done using Meyer's hematoxylin.

\section{Interpretation}

Positivity for p16 was considered when there was block staining of nuclear, along with or without cytoplasmic staining (19). The degree of intensity of the stain, parabasal involvement, pattern of staining (focal or diffuse), and percentage of positive staining dysplastic cells were analyzed. Each parameter was graded, and a combined score was used to determine positive or negative result using the criteria used by Alshenawy $\mathrm{H}$. et al. $(12,20)$ (Table 1). For p16 expression to be considered positive a total combined score of $>3$ was required.

Ki67 proliferation index is defined as the percentage of Ki67 positive cells. Grade 1+, 2+, and 3+ were given when the Ki67 index was below 5\%, 5-30\%, and greater than $30 \%$, respectively by observing nuclei of 200 epithelial cells located across the whole epithelial layer in high-power field as used by Alshenawy H. et al. (12).

Table 1. Immunohistochemistry scoring for p16

\begin{tabular}{|c|c|c|}
\hline \multicolumn{2}{|c|}{ p16 immunostaining grade: } & Score \\
\hline \multirow{4}{*}{ Percentage of positive cells (\%) } & $<5 \%$ & 0 \\
\hline & $5-49 \%$ & 1 \\
\hline & $50-80 \%$ & 2 \\
\hline & $>80 \%$ & 3 \\
\hline \multirow{4}{*}{ Intensity of reaction } & No reaction & 0 \\
\hline & Weak & 1 \\
\hline & Variable & 2 \\
\hline & Strong & 3 \\
\hline \multirow{3}{*}{ Cellular reaction pattern } & No reaction & 0 \\
\hline & Focal & 1 \\
\hline & Diffuse & 2 \\
\hline \multicolumn{3}{|c|}{ Total } \\
\hline \multicolumn{3}{|c|}{ Negative $(0-3)$} \\
\hline
\end{tabular}

\section{Statistical Analysis}

Data analysis was done using SPSS 17 (SPSS Inc., Chicago, Ill. USA); collected data was analysed by both descriptive and inferential methods. Descriptive method such as frequency and percentage were calculated to summarise the data. Sensitivity, specificity, positive predictive value and negative predictive value along with agreement were calculated.

\section{Results}

The age of the patients ranged between 28 and 68 years with majority of the patients being below 50 years of age. Majority of the cases of SIL were with parity of 2 or more. Most common clinical presentation was bleeding per vaginum. Of the total 31 cases, 12 were diagnosed as HSIL while 19 were diagnosed as LSIL on histopathology (Figure 1). Corresponding Pap smear was unavailable for 5 cases. The overall absolute correlation 
between cytology and histopathology was $58.1 \%$. HSIL was under-reported with 6 cases being reported out of 12 histopathologically diagnosed cases. Five ASC-H and one ASCUS assigned cases on cytology were upgraded to HSIL on histopathology. LSIL cases correlated well, with 13 out of 19 cases being assigned on cytology while one ASCUS case being upgraded to LSIL on histology. According to the criteria used by Alshenawy $\mathrm{H}$ et al., in the present study, $58.3 \%(7 / 12)$ cases of HSIL showed absolute $\mathrm{p} 16$ positivity while $26.3 \%(5 / 19)$ cases of LSIL showed p16 positivity (Figure 2 ).
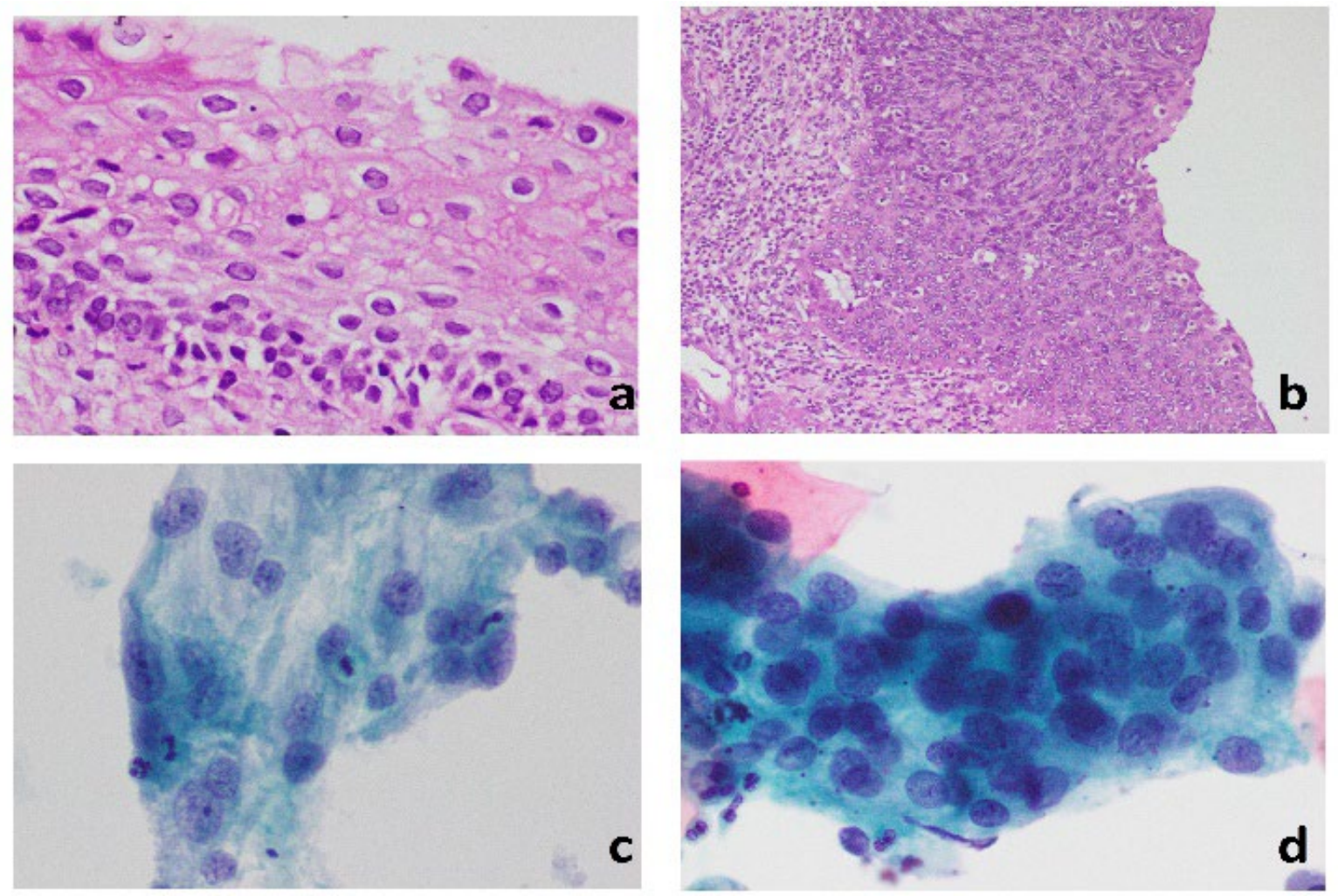

Fig 1. a: Low grade squamous intra-epithelial lesion (LSIL) H\&E 200X

b: High grade squamous intra-epithelial lesion (HSIL) H\&E 100X c: LSIL pap 400X d: HSIL pap 400X
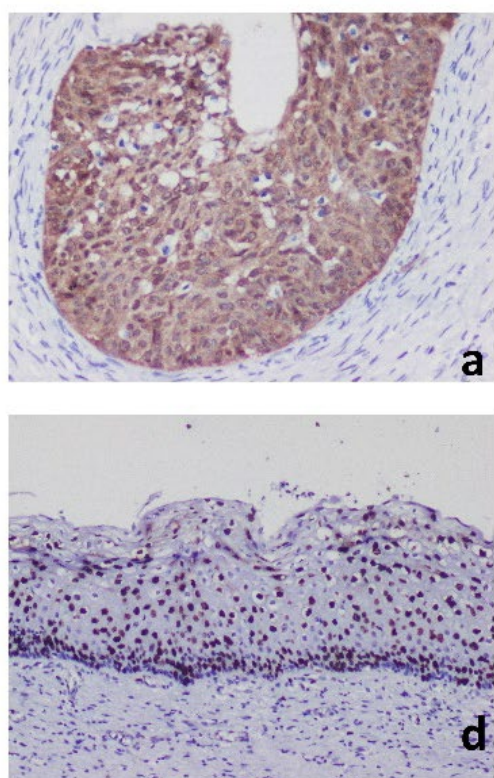
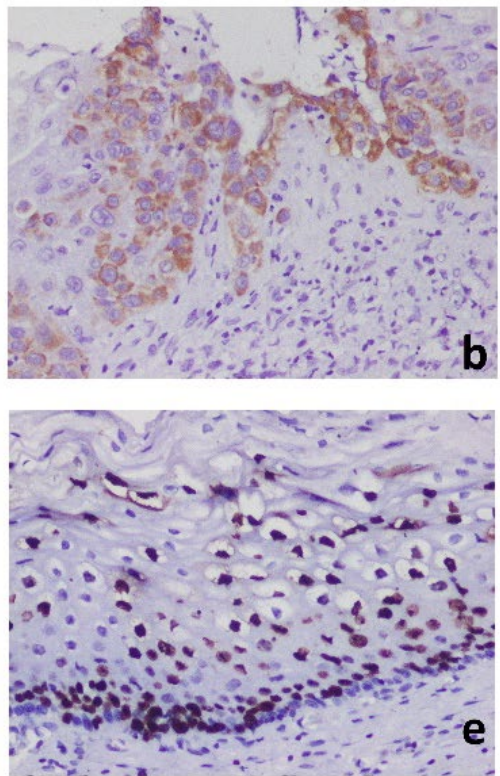
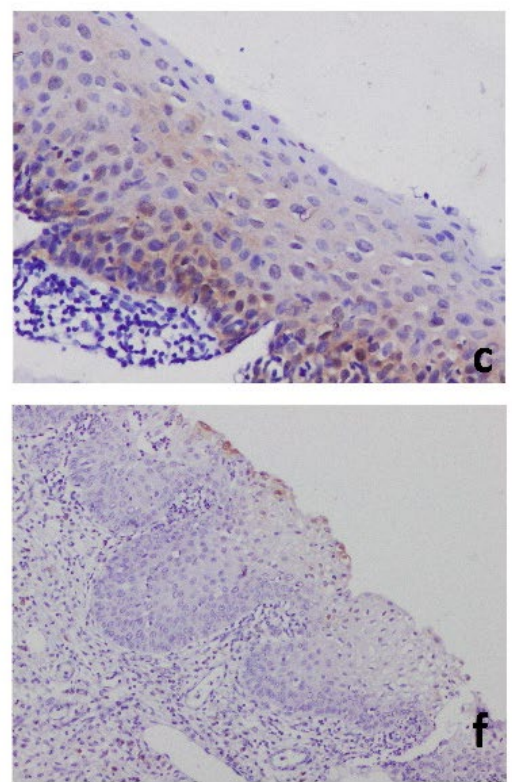

Fig 2. a:16 positive (score 8) with diffuse strong expression in HSIL,

b: p16 positive (score 4) with focal variable expression in HSIL c: p16 positive (score 4) with focal positive expression in LSIL d: Ki-67 grade 2+ in HSIL e: Ki-67 grade 2+ with full thickness expression in HSIL f: Ki-67 negativity in LSIL 
In the present study a total of $38.7 \%(12 / 31)$ cases of SILs showed absolute $\mathrm{p} 16$ positivity. Sensitivity of $\mathrm{p} 16$ was $38.7 \%$ with $100 \%$ specificity for SILs. Positive predictive value was $100 \%$ while negative predictive value was $51.3 \%$. Overall agreement between was 62.74 . Of 19 negative cases of p16, 4 cases showed absolute negativity with a score ' 0 '. While 15 cases had a score of 2 or more. Among all the SIL cases some amount of p16 activity was observed in $87 \%$ of the cases. The expression of Ki67 was "grade 1" in $67.7 \%$ of the cases with anatomical expression in the respective cases. The Ki67 expression in the middle and superficial third of the epithelium correlated well with the histopathological diagnosis with a P-value of 0.001 and 0.002 respectively. The control group comprising of diagnosed cases of chronic cervicitis showed no expression with p16 and with 5 out of 20 cases showing $<5 \%$ expression of Ki67 (Tables 2 and $\underline{3}$ ).

Table 2. p16 immunostain scoring in LSIL and HSIL

\begin{tabular}{|c|c|c|c|c|}
\hline \multicolumn{2}{|c|}{ p16 immunostaining } & HSIL n (\%) & LSIL n (\%) & Chronic Cx n (\%) \\
\hline \multirow{3}{*}{ Percentage of positive cells (\%) } & $0(<5 \%)$ & $5(41.7 \%)$ & $11(57.9 \%)$ & \multirow{3}{*}{$20(100 \%)$} \\
\hline & $1(5-49 \%)$ & $4(33.3 \%)$ & $6(31.6 \%)$ & \\
\hline & $2(50-80 \%)$ & $3(25.0 \%)$ & $2(10.5 \%)$ & \\
\hline \multirow{4}{*}{ Intensity of reaction } & 0 (No reaction) & $1(8.3 \%)$ & $3(15.8 \%)$ & \multirow{4}{*}{$20(100 \%)$} \\
\hline & 1(Weak) & $3(25.0 \%)$ & $10(52.6 \%)$ & \\
\hline & 2 (Variable) & $4(33.3 \%)$ & $4(21.1 \%)$ & \\
\hline & 3 (Strong) & $4(33.3 \%)$ & $2(10.5 \%)$ & \\
\hline \multirow{3}{*}{ Cellular reaction pattern } & 0 (No reaction) & $1(8.3 \%)$ & $3(15.8 \%)$ & \multirow{3}{*}{$20(100 \%)$} \\
\hline & 1 (Focal) & $10(83.3 \%)$ & $16(84.2 \%)$ & \\
\hline & 2 (Diffuse) & $1(8.3 \%)$ & $0(0 \%)$ & \\
\hline \multirow{3}{*}{ p16 Negative (0-3) } & 0 & $1(8.3 \%)$ & $3(15.8 \%)$ & \multirow{3}{*}{$20(100 \%)$} \\
\hline & 2 & $3(25.0 \%)$ & $7(36.8 \%)$ & \\
\hline & 3 & $1(8.3 \%)$ & $4(21.1 \%)$ & \\
\hline \multirow{4}{*}{ Positive (4-8) } & 4 & $4(33.3 \%)$ & $2(10.5 \%)$ & \\
\hline & 5 & $0(0 \%)$ & $2(10.5 \%)$ & \\
\hline & 6 & $2(16.7 \%)$ & $1(5.3 \%)$ & \\
\hline & 7 & $1(8.3 \%)$ & $0(0 \%)$ & \\
\hline
\end{tabular}

Table 3. Ki-67 grading in HSIL and LSIL

\begin{tabular}{|c|c|c|c|}
\hline Ki67 & HSIL n (\%) & LSIL n (\%) & Chronic Cx \\
\hline 0 & $0(0 \%)$ & $0(0 \%)$ & $16(80 \%)$ \\
\hline $1(<5 \%)$ & $6(50.0 \%)$ & $15(78.9 \%)$ & $4(20 \%)$ \\
\hline $2(5-30 \%)$ & $4(33.3 \%)$ & $4(21.1 \%)$ & $0(0 \%)$ \\
\hline $3(>30 \%)$ & $2(16.7 \%)$ & $0(0 \%)$ & $0(0 \%)$ \\
\hline
\end{tabular}

Table 4. Comparison of p16 and Ki67 expression between various studies and present study

\begin{tabular}{|c|c|c|c|c|c|c|c|}
\hline \multirow{2}{*}{ Reference } & n & \multicolumn{3}{|c|}{ LSIL } & \multicolumn{3}{c|}{ HSIL } \\
\cline { 2 - 9 } & & $\mathrm{n}$ & $\mathrm{p} 16$ & Ki67 & $\mathrm{n}$ & p16 & Ki67 \\
\hline Present study & 51 & 19 & 5 & 19 & 12 & 7 & 12 \\
\hline Hebbar et al. (13)2017 & 50 & 10 & 5 & 7 & 20 & 16 & 19 \\
\hline Alshenawy A et al. (8) 2014 & 75 & 15 & 4 & 6 & 48 & 32 & 48 \\
\hline Xing Y. et al. (19) 2017 & 95 & 45 & 11 & 16 & 40 & 35 & 38 \\
\hline Kanthiya K. et al. (16) 2016 & 243 & 106 & 11 & 24 & 61 & 48 & 46 \\
\hline
\end{tabular}

\section{Discussion}

Although histopathology remains the gold standard for the diagnosis of SILs, immune-histochemistry can be helpful in limited tissue biopsies and eliminating the interobserver variability. Recently p16 has gained popularity not only in typing the lesion but also in predicting treatment response (21). Several authors have studied p16 expression using different positivity criteria in both preinvasive and invasive squamous carcinomas 
(22-25). In this present study the grading used for p16 positivity, was proposed by Songkhun V. et al. and later used by Alshenawy H. et al. $(12,20)$.

In the present study the sensitivity for HSIL was $58.3 \%$ and for LSIL was $26.3 \%$. While other authors found p16 expression to be between $45 \%$ and $100 \%$ for HSIL and between $10 \%$ and $70 \%$ for LSILs (26). In the study by Eleuterio J. et al. in 2007 , a positive p16 reaction was seen in $92.3 \%$ cases and $15.4 \%$ cases of HSIL and LSIL respectively (27). Diane M.C. et al. observed $80.9 \%$ and $19.36 \%$ positivity with p 16 in HSIL and LSIL respectively (18). The authors also stated a sensitivity of $86.9 \%$ and a specificity of $87.7 \%$ with p 16 for HSIL (28). Srivastava S. et al. observed $100 \%$ positivity for p16 for both LSIL and HSIL (12). Xing Y. et al. observed $24.4 \%$ and $87.5 \%$ positivity with $\mathrm{p} 16$ in LSIL and HSIL respectively (29). In the study by Leite P. et al. p16 positivity observed was $12.8 \%$ and $72.1 \%$ for LSIL and HSIL respectively. They also observed a significant relation between $\mathrm{p} 16$ positivity and recurrence with P-value of 0.018 (30). Comparative analysis of $\mathrm{p} 16$ and $\mathrm{Ki} 67$ with various recent studies and the present study is depicted in Table 4.

The degree of dysplasia correlated with Ki67 expression, in most of the cases had grade 1 expression with $33.3 \%(4 / 12)$ cases of HSIL having grade 2 and $16.7 \%(2 / 12)$ cases of HSIL having grade 3 expression of Ki67. It was also observed that Ki67 expression was extending into the superficial third of the epithelial layer while LSIL demonstrated Ki67 expression which was limited to the basal third. These findings were similar to those by Srivastav S. et al. and Hebbar A. et al. $(22,23)$.

\section{Conclusion}

All the SILs showed 100\% Ki67 expression with a comparatively good expression of p16 in HSIL followed by LSIL. Such cases with positive p16 and high Ki67 expression should be further evaluated for HPV DNA typing as a routine protocol although not performed in the present study.

Sensitivity and specificity of p16 expression was low as compared to other studies. HPV infection status with p16 expression were not available due to resource constraints. Although we have considered block positivity for p16 as true positive, a uniform grading criterion for establishing p16 expression would be effective in interpretation with consensus.

\section{Acknowledgements}

The authors would like to express gratitude towards all who helped in the improvement of this paper.

\section{Conflict of Interest}

The authors confirm that there are no known conflicts of interest associated with this publication, and there has been no significant financial support for this work that could have influenced its outcome.
1. Bruni L, Barrionuevo-Rosas L, Albero G, Serrano B, Mena M, Gómez D, Muñoz J, Bosch FX, De Sanjosé S. ICO information centre on HPV and cancer (HPV information centre). Human papillomavirus and related diseases in the world. Summary Report. 2015 Mar;4(08).

2. Sreedevi A, Javed R, Dinesh A. Epidemiology of cervical cancer with special focus on India. Int J Womens Health. 2015;7:405-14. [DOI:10.2147/IJWH.S50001] [PMID] [PMCID]

3. Musa J, Achenbach CJ, O'Dwyer LC, et al. Effect of cervical cancer education and provider recommendation for screening on screening rates: A systematic review and meta-analysis [published correction appears in PLoS One. 2017 Dec 29;12 (12 ):e0190661]. PLoS One. 2017;12(9):e0183924. [DOI:10.1371/journal.pone.0183924] [PMID] [PMCID]

4. Khazaei S, Izadi B, Mirbahari SG, Madani SH, Malek Khosravi Sh, Emami Alagha M, Sajadimajd S. Comparison Between Two Detection Methods for HPV16, HPV18 and P16Ink4a Biomarkers in Diagnosis of Abnormal Cervical Cytology. Asian Pac J Cancer Prev. 2016 Dec 1;17(12):52237.

5. Solomon D, Nayar R. The Bethesda System for Reporting Cervical Cytology. Nayar R, Wilbur DC, editors. Cham: Springer International Publishing; 2015. 342.

6. Genovés J, Alameda F, Mancebo G, Solé JM, Bellosillo B, Lloveras B, et al. Human papillomavirus detection and p16INK4a expression in cervical lesions: A comparative study. Hum Pathol. 2014;45(4):826-33. [DOI:10.1016/j.humpath.2013.10.035] [PMID]

7. Galgano MT, Castle PE, Atkins KA, Brix WK, Nassau SR, Stoler MH. Using biomarkers as objective standards in the diagnosis of cervical biopsies. Am J Surg Pathol. 2010;34(8):1077-87.

[DOI:10.1097/PAS.0b013e3181e8b2c4] [PMID] [PMCID]

8. van Baars R, Griffin $\mathrm{H}, \mathrm{Wu} Z$, et al. Investigating Diagnostic Problems of CIN1 and CIN2 Associated With High-risk HPV by Combining the Novel Molecular Biomarker PanHPVE4 With P16INK4a. Am J Surg Pathol. 2015;39(11):1518-28. [DOI:10.1097/PAS.0000000000000498] [MID] [MCID]

9. de Freitas AC, Coimbra EC, Leitão MDCG. Molecular targets of HPV oncoproteins: Potential biomarkers for cervical carcinogenesis. Biochim Biophys Acta. 2014;1845(2):91-103. [DOI:10.1016/j.bbcan.2013.12.004] [PMID]

10. Wentzensen N, von Knebel Doeberitz M. Biomarkers in cervical cancer screening. Dis Markers. 2007;23(4):315-30. [DOI:10.1155/2007/678793] [PMID] [PMCID]

11. Rk M, Su V, Sk M, Nagger S, Shrivastava K, Mishra RK, et al. The use of molecular markers ( Ki67 \& p53) in premalignant and malignant cervical neoplasms. Trop J Pathol Microbiol. 2016;2(1):3-8. [DOI:10.17511/jopm.2016.i01.02]

12. Alshenawy HAS. Evaluation of p16, human papillomavirus capsid protein L1 and Ki-67 in cervical intraepithelial lesions: Potential utility in diagnosis and prognosis. Pathol Res Pract. 2014;210(12):916-21. [DOI:10.1016/j.prp.2014.07.007] [PMID]

13. Volgareva G, Zavalishina L, Andreeva Y, et al. Protein p16 as a marker of dysplastic and neoplastic alterations in cervical epithelial cells. BMC Cancer. 2004;4:58. [DOI:10.1186/1471-2407-4-58] [PMID] [PMCID]

14. Krishnappa P, Mohamad IB, Lin YJ, Barua A. Expression of P16 in high-risk human papillomavirus related lesions of the uterine cervix in a government hospital, Malaysia. Diagn

\section{References}


Pathol. 2014;9:202. [DOI:10.1186/s13000-014-0202-z] [PMID] [PMCID]

15. Sangwaiya A, Gill M, Bairwa S, Chaudhry M, Sen R, Prakash Kataria S. Utility of P16/INK4a and Ki-67 in Preneoplasticand Neoplastic Lesions of Cervix. Iran J Pathol. 2018;13(3):308-16.

16. Lee H, Lee H, Cho YK. Cytokeratin7 and cytokeratin19 expression in high grade cervical intraepithelial neoplasm and squamous cell carcinoma and their possible association in cervical carcinogenesis [published correction appears in Diagn Pathol. 2017 May 22;12 (1):40]. Diagn Pathol. 2017;12(1):18. [DOI:10.1186/s13000-017-0609-4] [PMID] [PMCID]

17. Kong J, Li Y, Liu S, et al. High expression of ezrin predicts poor prognosis in uterine cervical cancer. BMC Cancer. 2013;13:520. Published 2013 Nov 4. doi:10.1186/14712407-13-520. [DOI:10.1186/1471-2407-13-520] [PMID] [PMCID]

18. Zhang W, Ou J, Lei F, et al. C14ORF166 overexpression is associated with pelvic lymph node metastasis and poor prognosis in uterine cervical cancer. Tumour Biol. 2016;37(1):369-379. [DOI:10.1007/s13277-015-3806-3] [PMID] [PMCID]

19. Lim S, Lee MJ, Cho I, Hong R, Lim SC. Efficacy of p16 and Ki-67 immunostaining in the detection of squamous intraepithelial lesions in a high-risk HPV group. Oncol Lett. 2016;11(2):1447-52. [DOI:10.3892/ol.2015.4071] [PMID] [PMCID]

20. Vinyuvat S, Karalak A, Suthipintawong C, Kleebkaow P, Trivijitsilp P, Triratanachat $\mathrm{S}$, et al. Interobserver Reproducibility in Determining p16 Overexpression in Cervical Lesions : Use of a Combined Scoring Method. Asian Pac J Cancer Prev. 2008;9:653-7.

21. Schwarz JK, Lewis JS, Pfeifer J, Huettner P, Grigsby P. Prognostic Significance of p16 Expression in Advanced Cervical Cancer Treated With Definitive Radiotherapy. Int J $\begin{array}{lll}\text { Radiat } & \text { Oncol. } & 2012\end{array}$ [DOI:10.1016/j.ijrobp.2011.11.032] [MID]

22. Srivastava S. P16INK4A and MIB-1: An immunohistochemical expression in preneoplasia and neoplasia of the cervix. Indian $\mathrm{J}$ Pathol Microbiol. 2010;53(3):524. [DOI:10.4103/0377-4929.68301] [PMID]
23. Hebbar A, Murthy V. Role of p16/INK4a and Ki-67 as specific biomarkers for cervical intraepithelial neoplasia: An institutional study. J Lab Physicians. 2017;9(2):104. [DOI:10.4103/0974-2727.199630] [PMID] [PMCID]

24. Galgano MT, Castle PE, Atkins K a, Brix WK, Nassau SR, Stoler MH. Using Biomarkers as Objective Standards in the Diagnosis of Cervical Biopsies. Am J Surg Pathol. 2010 ;34(8):1077-87. [DOI:10.1097/PAS.0b013e3181e8b2c4] [PMID] [PMCID]

25. Eleutério Jr J, Lima T, Cunha M, Cavalcante D, Silva A. Immunohistochemical Expression of the Tumor Suppressor Protein p16INK4a in Cervical Adenocarcinoma. Rev Bras Ginecol e Obs / RBGO Gynecol Obstet. 2017;39(01):21-5. [DOI:10.1055/s-0037-1598042] [PMID]

26. Kanthiya K, Khunnarong J, Tangjitgamol S, Puripat N, Tanvanich S. Expression of the p16 and Ki67 in cervical squamous intraepithelial lesions and cancer. Asian Pacific J Cancer Prev. 2016;17(7):3201-6.

27. Eleutério Jr J, Giraldo PC, Gonçalves AK, Cavalcante DIM, Ferreira FVDA, Mesquita SM, et al. Prognostic markers of high-grade squamous intraepithelial lesions: the role of p16 INK4a and high-risk human papillomavirus. Acta Obstet $\begin{array}{llll}\text { Gynecol } & \text { Scand. } & 2007 & \text {;86(1):94-8. }\end{array}$ [DOI:10.1080/00016340601089727] [PMID]

28. Eleutério J, Cavalcante D, Linhares I, Pompeu M., Giraldo P. The utility of p16 INK4a and Ki-67 to identify high-grade squamous intraepithelial lesion in adolescents and young women. Indian J Pathol Microbiol. 2012;55(3):339. [DOI:10.4103/0377-4929.101740] [PMID]

29. Xing Y, Wang C, Wu J. Expression of geminin, p16, and Ki67 in cervical intraepithelial neoplasm and normal tissues. Medicine (Baltimore). $2017 \quad$;96(26):e7302. [DOI:10.1097/MD.0000000000007302] [PMID] [PMCID]

30. Leite P, Tafuri L, Costa M, Lima M, Simões R, Leite PM de $\mathrm{O}$, et al. Evaluation of the p16 and Ki-67 Biomarkers as Predictors of the Recurrence of Premalignant Cervical Cancer Lesions after LEEP Conization. Rev Bras Ginecol e Obs / RBGO Gynecol Obstet. 2017;39(06):288-93. [DOI:10.1055/s-0037-1598643] [PMID]

\section{How to Cite This Article}

Ghosh, A., Nirupama, M., Padmanabha, N., Kini, H. Assessment of p16 and Ki67 Immunohistochemistry Expression in Squamous Intraepithelial Lesion with Cytohistomorphological Correlation. Iranian Journal of Pathology, 2020; 15(4):268-273. doi: 10.30699/ijp.2020.112421.2208 